\begin{tabular}{lcr}
\hline \multicolumn{3}{c}{ A N N A L ES } \\
UNIVERSITATIS M A R I A E C URIE-SKŁ ODOWS K A \\
LOL. XXXVI & SECTIOFF & $1-2018$ \\
\hline
\end{tabular}

\author{
ANETA FILIPIUK-KUSZ \\ ORCID ID: https://orcid.org/0000-0003-4430-2441 \\ Uniwersytet Marii Curie-Skłodowskiej
}

\title{
Les modifications des locutions dans les commentaires sous les articles de presse en ligne (approche contrastive)
}

\begin{abstract}
Modyfikacje związków frazeologicznych w komentarzach do artykułów prasowych w Internecie (analiza porównawcza) Modifications in idiomatic expressions in comments from Internet editions of press texts (contrastive approach)
\end{abstract}

\section{INTRODUCTION}

L'Internet est un lieu de communication avec l'accès libre aux discussions pour tous les gens. Les locuteurs y expriment leurs opinions, sentiments, et réagissent aux commentaires des autres. Ils cherchent à «donner du sens» à la rupture de l'ordre normal des choses, à le réduire et dissoudre (Veniard, 2013: 7). Les forums deviennent intéressants grâce à la présence des expressions figées et par la concentration des mots employés à l'oral. Dans le présent article nous voulons décrire les dimensions formelle, sémantique et pragmatique des phraséologismes employés dans les commentaires sous les articles de presse en polonais et en français. Certains utilisateurs profitent de la richesse de la langue et commentent des événements importants en employant les séquences figées pourvues d'une valeur expressive. Notre analyse est fondée sur un corpus de quotidiens français et polonais en ligne ${ }^{1}$. Elle se concentrera en principe sur le langage standard et courant, puisque le lexique qui apparaît habituellement dans les usages quotidiens est employé de manière naturelle dans les forums de discussion. D’abord, nous

${ }^{1}$ Le corpus a été tiré de quotidiens et de revues tels que: Le Monde, Le Point, Le Figaro, Gazeta Wyborcza, Rzeczpospolita et des sites d'Internet. 
allons présenter les caractéristiques de la langue d'Internet et préciser des outils méthodologiques, à savoir certaines notions utilisées en phraséologie telles que figement, défigement, détournement, locution pour passer ensuite à la typologie des modifications selon différentes approches.

L'espace virtuel nous offre une immense variété de modes de communication qui peuvent être subdivisés d'après des critères différents. Nous allons décrire les usages des lexiques utilisés dans les forums de débats par les intervenants provenant de divers milieux sociaux afin d'en déterminer les spécificités. Nous nous intéressons en particulier à la diversité des lexiques employés dans les commentaires sous les articles de presse en ligne.

\section{QUELQUES CARACTÉRISTIQUES DE LA LANGUE D'INTERNET}

Le lexique qui apparaît habituellement dans les usages quotidiens est employé aussi dans les forums de discussions. C'est une propriété d'un langage standard et courant. Dans les textes produits par les internautes, on peut repérer les mots appartenant à différents registres de la langue : argotique, populaire, courant et familier. La communication virtuelle englobe les situations particulières où les utilisateurs proviennent de divers milieux sociaux et ils sont influencés par diverses cultures.

Nous pouvons noter les usages de mots non-conventionnels comme par exemple des mots populaires ou vulgaires. On distingue deux types de communication sur Internet: unilatéral et interactif. Dans le premier cas, l'expéditeur n'attend pas de réponse et n'interagit pas avec d'autres personnes. La communication englobe les bases de données, les écritures électroniques, le site WEB, l'assistance et les manuels des programmes. Le deuxième type, c'est la communication interactive où deux interlocuteurs participent à la conversation. Aouil (2008: 15) parle aussi de la communication synchrone lorsque les réactions d'un destinateur sont suspendues dans le temps (les forums de discussions, le courriel), et de la communication asynchrone qui se déroule dans le temps réel (les chats ou la messagerie instantanée).

Selon Le Nouveau Petit Robert (2009), le forum de débat est un «espace virtuel consacré à l'échange de messages écrits, aux discussions sur un thème, en temps différé entre utilisateurs d'un réseau télématique».

Ce genre de la communication synchrone se caractérise par:

- pratiques scripturales développées,

- thème du débat: politique, philosophique ou socioculturel,

- les participants à la discussion choisissent souvent un code graphique plus correct que dans un forum de discussion (Lazar, 2016: 43). 
À notre connaissance, l'analyse qualitative du contenu des commentaires sur les sites d'information en ligne à laquelle nous nous intéressons n'a pas encore fait l'objet de travaux approfondis. Le terme commentaire est entendu ici dans le sens propre du terme comme un ensemble des explications, des remarques, des observations faites à propos d'un texte. Dans notre étude nous focaliserons notre attention sur l'un des aspects du lexique utilisé dans les commentaires, à savoir sur l'emploi des locutions figées et leurs modifications.

\section{PROCÉDÉ DE FIGEMENT ET DE DÉFIGEMENT}

Dans ce qui suit, nous nous penchons sur la question des manipulations des locutions dans les commentaires en ligne. La définition de la locution que nous adoptons est celle de Gross (1996: 154):

[La locution est] un syntagme (nominal, verbal, adjectival, adverbial) dont les éléments composants ne sont pas actualisés individuellement et qui forme un concept autonome, que le sens global soit figé ou non. On parlera aussi de catégorie complexe ou polylexicale.

Cette unité polylexicale refuse certaines transformations syntaxiques normalement acceptables dans le cas des séquences libres comme par exemple la passivation, la relativisation, le détachement, la pronominalisation, l'extraction (Gross, 1996: 12-13). En revanche, la locution qui admet différentes transformations est une unité défigée. Schapira (1999), qui se sert du terme détournement, soutient que ce procédé est une manœuvre lexicale, sémantique ou stylistique, à la suite de laquelle une expression figée acquiert un sens discursif. Le détournement peut se manifester à travers :

- un détournement lexical qui consiste à remplacer «un (ou plusieurs éléments) lexicaux par un autre au niveau lexical, ou à insérer d'autres éléments, étrangers à la formule» (Schapira, 1999: 147),

- un détournement sémantique lorsque la locution «citée dans son sens littéral»ou «la locution, manipulée ou laissée intacte, est occasionnellement détournée de son sens premier soit par un contexte très différent de celui de sa première énonciation, soit par une situation de discours sans rapport avec son emploi habituel» (Schapira, 1999: 148-150).

Il est important de noter que les variantes ou les unités détournées peuvent être créées à partir des locutions et des proverbes canoniques. Dans le cas des variantes le sens original est conservé, par contre il est totalement différent dans les locutions et proverbes détournés (Woźniak, 2009: 187). 
En ce qui concerne le défigement, ce procédé, conçu en tant que «toute atteinte à la fixité formelle et à la globalité sémantique de séquence figée» (Mejri, 2009: 158-160) se manifeste à travers: le défigement relevant de la fixité formelle (de nature phonétique, morphologique, paradigmatique, syntagmatique, d'actualisation et syntaxique, de la combinatoire discursive) et celui de la fixité sémantique (dualité littéral/global, remotivation du sens littéral par association, remotivation par mention métalinguistique).

Ce phénomène linguistique a été aussi décrit par Grésillon et Maingueneau (1984 : 116) qui ont distingué, à l'intérieur du proverbe, les modifications suivantes :

- la modification d'un phonème (À chaque jour suffit sa peine - À chaque jour suffit sa veine);

- la modification d'un terme: À chaque jour suffit sa peine-À chaque nuit suffit sa peine;

- le fait d'adjoindre un élément: L'homme propose, Dieu dispose-L'homme propose, Dieu dispose, femme impose;

- la négation: Qui dort dîne - Qui ne dort pas dîne;

- la fusion des proverbes: À chaque jour suffit sa peine/À quelque chose malheur est bon - À chaque jour malheur est bon.

Lecler (2007: 48) note pour sa part quatre types de manipulations: la substitution, l'ajout d'un élément lexical, l'effacement du trait d'union, l'imbrication d'une seconde expression figée.

\section{ANALYSE DES CAS}

En français, les expressions figées utilisées dans les discussions spontanées sur les forums appartiennent à différents registres de langue: argotique, populaire, vulgaire (avoir du cul), familier (faire caca), courant (retourner la langue dans sa bouche), standard (faire long feu). Parmi les procédés fréquemment employés dans les commentaires en ligne, on peut mentionner:

- ajout (Le bouc émissaire de tous les médias du monde entier);

- substitution (Retournez donc dix fois la langue dans votre bouche avant de sortir des inepties);

- jeux de mots fondés sur la paronymie (Après Fillon, un autre filon!), ou sur les deux niveaux de la signification - un sens compositionnel et un sens global (Allez, dormez en paix, bonnes gens).

Dans tous ces cas, les compétences linguistiques des locuteurs garantissent le décodage des effets de sens (Ben Amor, 2008). 
Dans ce qui suit, nous allons étudier successivement divers types de manipulation appliqués aux expressions en français et en polonais.

\subsection{L'ajout d'un élément}

Cette opération apparaît assez fréquemment dans nos deux corpus d'exemples. Un constituant ajouté crée une modification et le sens global de la séquence canonique serrés comme des sardines ('entassés dans un espace restreint sans jouir d'aucune liberté de mouvements; très serrés') se trouve alors amplifié:

[....] c'est peut être pour cette raison que l'on peut apercevoir sur le bord des routes des tuperwares blancs serrés et empilés comme des sardines. (Posté le avril 18)²

Jesteśmy ściśnięci jak sardynki, duża sala, samorządy, zarządy, startujący w olimpiadach, zespół redakcyjny z Michaliną, ludzie pisujący do TW [...]. (NKJP, consulté le 10.02.2018)

[Nous sommes serrés comme des sardines, une grande salle, des autorités municipales, des conseils d'administration, les personnes participants à des concours et des compétitions olympiques, une équipe éditoriale avec Michalina, des gens qui écrivent pour TW.]

Le français et le polonais admettent les variantes avec l'expansion (en boîte) $w$ puszce) qui concrétise le sens global des deux séquences en mettant en relief leur motivation.

Manche: les parents dénoncent des classes surchargées et des élèves serrés «comme des sardines en boîte». (www.lamanchelibre.fr, consulté le 3.02.2017)

W wagonie było cicho, nikomu z tych ściśniętych jak sardynki w puszce ludzi nie chciało się gadać. (NKJP, consulté le 10.02.2018)

[Il y avait du silence dans la voiture, aucun des passagers serrés comme des sardines en boîte ne pouvait parler.]

En français serrés comme des sardines est une version moderne de serrés comme des harengs en caque, tandis qu'en polonais on note aussi la forme plus ancienne avec la variation de l'élément verbal gnieść, tłoczyć się, być stloczonym, ściśniętym jak śledzie w beczce (Stownik frazeologiczny PWN, 2007 : 469):

Qui construit maintenant ces énormes radeaux pneumatiques ???? [...] Maintenant tassés comme des harengs en caque, ils téléphonent à Bruxelles avant l'appareillage pour être pris en sauvetage sur leur boudins gonflables qui j'espère sont détruits ensuite. (www.lefigaro.fr, 19.12.2016)

Codziennie rano jest to samo. Dwa, maksymalnie trzy wagony i pełno ludzi. Jedziemy ściśnięci jak śledzie. Pociągi są nowe, ale co z tego, skoro w takich warunkach o wygodzie trudno mówić. (www.pszczyńska.pl, consulté le 27.11.2017)

${ }^{2}$ Http://campingcarpoidslourd.com/page/6/?attachment_id=irzhbzob (11.01.2018). 
[Chaque matin est le même. Deux ou trois voitures au maximum, pleines de gens. Nous nous déplaçons en métro, serrés comme des harengs. Les trains sont neufs, mais il est difficile de parler du confort dans telles conditions.]

\subsection{Modifications morphosyntaxiques}

a) la négation

Pas être serrés comme des sardines en boîte

Vendredi, de manière très symbolique, une banderole, réalisée par un parent d'élève, a été hissée en contestation et en poursuite des actions «les enfants ne doivent pas être serrés comme des sardines en boîte». (www.ouest-france.fr, consulté le 12.02.2018)

b) la mise au singulier

Serré comme une sardine

«C'était horrible», raconte Maxime, un usager quotidien de la ligne qui travaille près de la station Charles de Gaulle Étoile. Lundi soir, la coulée de boue accidentelle sur les voies a eu raison de lui. Exaspéré par la situation, il renonce à prendre le métro ou le RER et commande un VTC, persuadé que le trafic reviendrait à la normale d'ici le lendemain matin, «mais pas du tout». Pour se rendre au travail, il tente le RER de Bry-sur-Marne jusqu'à Vincennes. Mais avec un train toutes les 20 minutes, il n'est qu'au début de ses peines. «J'en ai laissé passer trois. Et quand je suis finalement monté, j'étais serré comme une sardine». (www.lexpress.fr, le 31.10.2017, consulté le 3.02.2018)

[...] kiedy rozpędzą konie, kibitka się wywraca i konie wleką po ćwierć mili, a ja zamknięty tluke się jak śledź w beczce [...]. (www.wyborcza.pl, consulté le 21.09.2001)

$[(\ldots)$ quand les chevaux se précipitent, le chariot se renverse, et puis, ils traînent un quart de mile, et moi, enfermé, je me sens comme un hareng dans un tonneau (...).]

La forme et le contenu sémantique des locutions sont touchés: il s'agit de préciser le sens global des deux phraséologismes et, en même temps, d'animer leur sens propre.

\subsection{La substitution}

On distingue divers types de variations (Bernet, 1992):

- les variations qui consistent à substituer un élément à un autre (bête de scène 'artiste qui a beaucoup de présence' au lieu de bête de cimes),

- les variations synonymiques et parasynonymiques qui peuvent être lexicalisées ou idiolectales et qui s'appuient sur l'organisation interne du lexique propre aux langues naturelles, 
- les variations à l'intérieur de classes distributionnelles, ce sont les termes qui se substituent et qui ont le même sème générique (le même «genre prochain»), par exemple: avoir un métro / une rame / un train de retard (genre prochain: transports en commun ferroviaires), "être à la traîne»,

- les variations distributionnelles dans des classes ouvertes.

a) la substitution d'un élément nominal par son synonyme

La substitution consiste alors à substituer un constituant nominal de la locution par son synonyme expressif: mettre la main / la patte sur quelque chose / quelqu'un ('s'emparer de, étendre son pouvoir à'). La forme canonique n'est pas stylistiquement marquée, ce qui est attesté dans la plupart des dictionnaires ( $L e$ Nouveau Petit Robert, 2009; Rey Chantreau, 1993; TLFi). Il est intéressant de noter qu'en français la variante avec son synonyme expressif mettre la patte sur apparait dans TLFi' ${ }^{3}$.

Voyons maintenant de plus près l'exemple suivant:

Mettre sa patte sur un meuble de designer

Design Bespoke propose, sur Internet, des pièces signées de créateurs comme Jean Nouvel, mais personnalisables. Une petite révolution.

C'est le coup de foudre. Le canapé, la table ou la bibliothèque design dont on rêvait existe bel et bien... mais ses dimensions ou sa teinte se marient mal avec le salon. Comme il est plus aisé d'adapter son mobilier à son intérieur que de déménager, Bespoke, nouveau venu dans l'édition de meubles, a développé un concept original: des pièces pensées par des designers, mais personnalisables. Dimensions (au centimètre près), couleurs et parfois matériaux peuvent varier, permettant à l'acheteur de créer un meuble unique. (www.24heures.ch, consulté le 12.09.2015)

La locution a été insérée ici dans un contexte lexical spécifique, ce qui anime son sens littéral. Par conséquent, les deux sens coexistent dans l'esprit du locuteur lors du décodage du message. Il semble que le mot patte ne véhicule pas de connotations péjoratives, mais, au contraire, positives liées à une ambiance de familiarité entre le vendeur et le client.

En polonais, le constituant nominal łapa ('patte') appliqué à un humain a un sens péjoratif (Tokarski, $1993: 359$ ):

Tę wiedzę o wszystkich ma tylko paru najważniejszych funkcjonariuszy, którzy zdołali przeniknąć do rządu Olszewskiego lub kancelarii Wałęsy i położyć łapę na aktach służb, które nie zostały zniszczone. (www.wyborcza.pl, consulté le 2.11.2017)

[Seuls quelques officiers les plus importants ont cette connaissance de tous ceux qui ont réussi à pénétrer dans le gouvernement de Olszewski ou le bureau de Wałęsa et à mettre la patte sur les dossiers des services spéciaux qui n'ont pas été détruits.]

${ }^{3}$ Selon le TLFi 'se saisir de quelqu'un ou de quelque chose pour se l'approprier de manière exclusive; découvrir ce qu'on cherchait'. 
b) la substitution d'un élément verbal

La locution canonique jeter le bébé avec l'eau du bain, enregistrée dans les dictionnaires français, signifie 'perdre de vue l'essentiel ou se débarrasser d'une chose pourtant importante dans le but d'éliminer avec les ennuis ou contraintes qu'elle implique'. Elle a pour des équivalents en polonais deux formes: wylać dziecko z kapiela /nie wylewać dziecka z kapiela ('supprimer quelque chose d'important en faisant quelque chose d'autre'). En français, les termes se substituent à l'intérieur d'une même classe distributionnelle:

Eurotunnel, la France et la G-B se renvoient le bébé avec l'eau du bain... bravissimo! (www. lefigaro.fr, consulté le 29.07.2015)

Cette locution possède plusieurs variantes: jeter/rejeter/renvoyer le bébé avec l'eau du bain, et ce fait est un indice qu'on a affaire aux formes pas encore lexicalisées ou idiolectales.

En polonais, on a deux formes qui coexistent:

Wylewanie dziecka z kapiela - WikiLeaks i zasada Prawdy

Jest takie stare powiedzenie, żeby „,nie wylewać dziecka z kapiela”, i nigdy nie było ono bardziej adekwatne niż w obecnej sytuacji związanej z WikiLeaks i Julianem Assange'em ${ }^{4}$.

[Jeter le bébé avec l'eau du bain (dosł. verser) - WikiLeaks et le principe de la vérité

Il y a un vieux dicton qui dit «ne pas jeter le bébé avec l'eau du bain», et il n'a jamais été plus adéquat que dans la situation actuelle liée à WikiLeaks et Julian Assange.]

Jestem przewodnikiem turystycznym, pracuję na umowy o dzieło, w ubiegłym roku PIT-y otrzymałem od 48 zleceniodawców i mam trafić do wora razem z przekręciarzami „,przerabiającymi” etaty na pasmo umów o dzieło? Znów możemy „wylać dziecko z kapiela””. (www.rzeczpospolita.pl, consulté le 28.01.2014)

[Je suis un guide touristique, j'ai un contrat de travail temporaire, l'année dernière j'ai reçu des PIT de 48 clients, et par conséquent je serai parmi les escrocs qui «remplacent» un poste à durée indéterminée par une série de contrats temporaires. Encore une fois, nous pouvons «jeter le bébé avec l'eau du bain»»]

\subsection{Croisements de formes}

Il est intéressant que parmi les opérations utilisées dans le discours journalistique, on trouve aussi celles basées sur les jeux de mots, ceux-ci étant entendus comme des contaminations de sens et croisements de formes (Guiraud, 1962: 85).

a) au niveau phonétique

Les jeux de mots sont ici fondés sur l'homophonie qui, manipulant des sonorités, crée deux énoncés homophones (le plus souvent humoristique) : j'en ai marre

${ }^{4}$ https://pracownia4.wordpress.com/2010/12/11/wylewanie-dziecka-z-kapiela-\%E2\%80\%93wikileaks-i-zasada-prawdy/ 
('j’en ai assez, je suis excédé, écœuré'); c'est marre! ('c'est assez, cela ne doit plus continuer ainsi') et $j$ 'en neymar ${ }^{5}, j$ 'en Aymar:

J'en neymar jusqu'à la nausée. (Le Point, consulté le 02.08.2017)

Le Barça, le PSG, le Qatar, depuis des jours ...ouf...

J'aime bien le foot mais là: j'en Aymar. (www.lefigaro.fr, consulté le 04.08.2017)

J'en ai marre de ce Neymar. (www.lefigaro.fr, consulté le 04.08.2017)

J'en Naymar de tout ce tapage autour de ce footeux !! tout ce fric c'est écœurant. (www.lefigaro. fr, consulté le 03.08.2017)

J'en ai marre et c'est marre! ont pour des équivalents en polonais des expressions telles que : ktoś ma czegoś po dziurki w nosie / po uszy / ktoś ma dość czegoś. Leur accumulation dans le texte a pour effet de marquer l'hyperbole et l'ironie, ainsi que d'exprimer l'état émotionnel du locuteur:

Miałem dość, jako człowiek, po uszy i po dziurki w nosie całej atmosfery. Jak oddawałem, to było źle, choć to jedyna możliwość jak najszybszego uwolnienia się od tej przeklętej zarazy, którą jest dekret - mówi Rudnicki. - Jak nie oddawałem, było jeszcze gorzej. (www.warszawa.wyborcza. pl, consulté le 25.01.2014)

[J'en avais vraiment assez, j'en avais marre de toute cette atmosphère. Quand je rendais ce décret, c'était mauvais, même si c'était le seul moyen de me libérer de «ce fléau maudit» - disait Rudnicki. - Quand je ne le rendais pas, c'était encore pire.]

b) au niveau du sens étymologique et global

Dans :

Faire tout le contraire de ce qu'il souhaite, c'est une belle occasion de lui faire un bras d'honneur à celui qui a déshonoré la France! (www.lemonde.fr, consulté le 29.04.2017)

le décodage du message est conditionné par les facteurs socio-culturels et les compétences linguistiques de l'interlocuteur. D'une part, faire un bras d'honneur renvoie globalement à un geste vulgaire et obscène qui exprime le profond mépris ressenti à l'égard de l'individu à qui on l'adresse, d'autre part, on reconnaît deux mots qui, du point de vue sémantique sont des antonymes : honneur et déshonorer.

\section{EN GUISE DE CONCLUSION}

Dans le discours journalistique, les manipulations des locutions peuvent se manifester à l'aide de divers procédés. La classification de ceux-ci n'est pas totalement

${ }^{5}$ Neymar est un footballeur brésilien, depuis 2017 il joue pour le Paris Saint-Germain, il est devenu le joueur le plus cher de l'histoire du football, son transfert a été fortement exploité dans les médias français. 
claire et facile à construire vu que chaque manipulation est un fait singulier. Les opérations décrites, employées volontairement par les internautes, modifient souvent la structure et le sens des locutions. Elles ont pour but d'attirer l'attention des locuteurs: l'effet ludique vient des jeux réalisés sur le plan phonétique, formel et sémantique. Il est à noter que certaines variations peuvent être idiolectales.

Notre étude montre que les mêmes modifications des locutions se manifestent en polonais et en français : l'adjonction et la substitution d'éléments ou les jeux de mots permettant d'activer un sens littéral ou un sens nouveau actualisé dans un contexte adéquat. Les opérations caractéristiques pour le français sont la négation ou le croisement des formes au niveau du sens étymologique et global.

\section{RÉFÉRENCES BIBLIOGRAPHIQUES}

Aouil, B. (2008). Komunikowanie się w Internecie - narzędzia, specyfika i właściwości. In: M. Wawrzak-Chodaczek (ed.), Komunikacja społeczna w świecie wirtualnym (pp. 10-40). Toruń: Wydawnictwo Adam Marszałek.

Ben Amor Ben Hamida, T. (2008). Défigement et traduction intralinguale et interlinguale. Meta: Journal des Traducteurs, 53(2), 443-455.

Bernet, Ch. (1992). Sur quelques expressions du français populaire d'aujourd'hui et leurs variantes. Grammaire des fautes et français non conventionnel. Paris: Presses de l'École Normale Supérieure.

Burbea, G. (2013). Les jeux de mots - déconstruction et reconstruction de sens. Studia Romanica Posnaniensia, 40(1), 149-157.

Grésillon, A., Maingueneau, D. (1984). Polyphonie, proverbe et détournement. Langages, 73, 112-125.

Gross, G. (1996). Les expressions figées en français. Noms composés et autres locutions. Paris: Ophrys.

Guiraud, P. (1962). Les locutions françaises. Paris: Que sait-je?

Kłosińska, A. (2007). Słownik frazeologiczny PWN. Warszawa: WN PWN.

Lazar, J. (2016). À propos des pratiques scripturales dans l'espace virtuel: entre Facebook et Twitter. Ostrava: Universitas Ostraviensis.

Lecler, A. (2007). Le défigement: un nouvel indicateur des marques du figement? Cahiers de Praxématique, 45, 43-60.

Majkowska, G. (1988). Klasyfikacja semantyczna zamierzonych modyfikacji. In: M. Basaj, D. Rytel (red.), Z problemów frazeologii polskiej i stowiańskiej, 5 (pp. 143-163). Wrocław: Zakład Narodowy im. Ossolińskich.

Mejri, S. (2009). Figement, défigement et traduction. Problématique théorique. In: S. Mejri, P. Mogorrón Huerta (eds.), Figement, défigement et traduction. Rencontres méditerranéennes, 2 (pp. 153-163). Alicante: Universidad de Alicante.

Rey, A., Chantreau, S. (1989). Dictionnaire des Expressions et Locutions. Paris: Le Robert.

Rey, A., Rey-Debove, J. (eds). (2009). Le Nouveau Petit Robert de la langue française. Paris: Le Robert.

Schapira, Ch. (1999). Les stéréotypes en français. Les proverbes et autres formules. Paris: Ophrys.

Skorupka, S. (1989). Stownik frazeologiczny języka polskiego. Warszawa: Wiedza Powszechna.

Tokarski, R. (1993). Słownictwo jako interpretacja świata. In: J. Bartmiński (red.), Współczesny język polski. Encyklopedia Kultury Polskiej XX Wieku, 2 (pp. 335-362). Wrocław: Wiedza o kulturze. 
Veniard, M. (2013). Les nominations des événements dans la presse. Essai de sémantique discursive. Paris: Presse Universitaire de Franche-Comté.

Woźniak, A. (2009). Le proverbe détourné : étude théorique appliquée à un corpus bilingue francoespagnol. Paremia, 18, 185-196.

\title{
Sitographie
}

http://www.presseelectronique.com/france/pressefrancaise.htm (11.01.2018).

http://www.journauxfrancais.net/ (11.01.2018).

TLFi - Trésor de la Langue Française informatisé http://atilf.atilf.fr/tlf.htm (11.01.2018).

\section{RÉSUMÉ}

Dans cet article, nous étudions les différents types de modifications des unités phraséologiques qui apparaissent dans les textes de presse électronique française et polonaise et les commentaires sous les articles en ligne. Notre objectif principal était de décrire le fonctionnement de ces unités dans le discours journalistique et de montrer la diversité des procédés utilisés en français et en polonais. Notre analyse a permis de détecter les opérations communes et celles qui sont caractéristiques pour les deux langues comparées. Certaines modifications ont pour effet les jeux de mots permettant d'activer un sens littéral ou un sens nouveau actualisé dans un contexte adéquat.

Mots-clés : unité phraséologique, texte de presse, différents types de modifications, jeux de mots

\begin{abstract}
The paper presents the analysis of types of modifications in idiomatic expressions based on the particular examples extracted from comments and opinions from press texts in both Polish and French Internet editions. The main task of the analysis was to reveal the diversity of the operations and to compare how they function in both languages. The review of the examples from the presented phraseological innovations proves that both common as well as different operations exist for both Polish and French. Some modifications have the effect of word games allowing to activate a literal sense or a new meaning in a context.
\end{abstract}

Keywords: idiomatic expression, press text, types of modifications in idiomatic expressions, word games

\begin{abstract}
ABSTRAKT
W artykule przeanalizowano pod różnym kątem przykłady modyfikacji związków frazeologicznych, pochodzące z komentarzy i wypowiedzi do tekstów prasowych w polskich i francuskich wydaniach internetowych. Głównym zadaniem było pokazanie różnorodności użytych środków oraz porównanie sposobu ich funkcjonowania w obu językach. Analiza przykładów innowacji frazeologicznych dowodzi, że istnieją operacje zarówno wspólne, jak i odmienne dla obu systemów językowych. W niektórych modyfikacjach występuje gra słów, w której aktualne staje się znaczenie dosłowne związku lub w odpowiednim kontekście pojawia się nowe znaczenie.
\end{abstract}

Słowa kluczowe: związek frazeologiczny, tekst prasowy, typy modyfikacji frazeologicznych, gra słów 
